\title{
Capturing Movement Decomposition to Support Learning and Teaching in Contemporary Dance
}

\author{
JEAN-PHILIPPE RIVIĖRE, SARAH FDILI ALAOUI, BAPTISTE CARAMIAUX, and WENDY \\ E. MACKAY, University Paris-Sud, CNRS, Inria,France
}

Our goal is to understand how dancers learn complex dance phrases. We ran three workshops where dancers learned dance fragments from videos. In workshop 1, we analyzed how dancers structure their learning strategies by decomposing movements. In workshop 2, we introduced MoveOn, a technology probe that lets dancers decompose video into short, repeatable clips to support their learning. This served as an effective analysis tool for identifying the changes in focus and understanding their decomposition and recomposition processes. In workshop 3, we compared the teacher's and dancers' decomposition strategies, and how dancers learn on their own compared to teacher-created decompositions. We found that they all ungroup and regroup dance fragments, but with different foci of attention, which suggests that teacher-imposed decomposition is more effective for introductory dance students, whereas personal decomposition is more suitable for expert dancers. We discuss the implications for designing technology to support analysis, learning and teaching of dance through movement decomposition.

CCS Concepts: • Human-centered computing $\rightarrow$ Empirical studies in HCI; Empirical studies in collaborative and social computing.

Additional Key Words and Phrases: Learning Dance; Technology Probe; Qualitative Methods; Movement Decomposition

\section{INTRODUCTION}

Contemporary dance is a corporal practice that undoubtedly requires motor skills of the highest level. To reach expert level, dancers are trained to efficiently acquire complex movements and phrases. In a dance class, teachers or choreographers guide students through movement phrases until proficiency is achieved. In this context, teachers and choreographers use strategies to facilitate movement transmission to the dancers which include demonstrations, movement decomposition and repetition. Outside of a dance class, dancers are often led to learn dance phrases through video recordings. For example, the state diploma of dance teacher training in France requires students to learn dance phrases from video recordings ${ }^{1}$ that they have to perform on the examination day. In this context, dancers learn to transpose movements from a video to physical movements. In both cases, movement transmission (either from video to dancers or from teacher to dancers) is mostly based on heuristics and habits from personal practice, the dance community and dance pedagogy. Digital technology has barely been explored as a means to better understand dance learning practice and to facilitate dance movement learning in the long run.

Supporting movement learning fundamentally involves better understanding the role of practice. Indeed, while complex movement skill acquisition remains not fully understood, there is agreement that practice has a central role [19]. By repeating and varying a movement to be learned, a dancer acquires it, meaning that he or she will able to perform it faster and more accurately [15]. Since practice is so central to the acquisition of any motor skill, the factors of good practice have been widely investigated in the literature. For example, the way task variations are scheduled within practice sessions can have a significant impact on motor learning [8, 32, 37]. However, very little

\footnotetext{
${ }^{1}$ Examen d'Aptitude Technique (EAT) http://www.culture.gouv.fr/Thematiques/Danse/Enseignement-formation-et-metiers
}

Authors' address: Jean-Philippe Rivière, riviere@lri.fr; Sarah Fdili Alaoui, saralaoui@lri.fr; Baptiste Caramiaux, caramiaux@ lri.fr; Wendy E. Mackay, mackay@lri.fr, University Paris-Sud, CNRS,, Inria, F-91400, Orsay, France. 
is known about how practice is implemented by dancers in a classroom while interpreting the teacher's instructions or when learning on their own from videos.

The challenge stems from the tacit aspects of the process: learning new motor skills is partially tacit and not conscious to the learner [29]. For example, the strategies used by a dancer when learning a new movement are, to some extent, driven by the dancer's embodied knowledge, the type of knowledge that the body constructs and produces implicitly [41]. Movement acquisition critically relies on this embodied knowledge as well as the dancer's prior skills. Additionally, through dance training, dancers have acquired practice techniques that help them to practice movement in diverse ways. An example of such a technique is marking, which is a way to practice a reduced form of the movement by emphasizing its key elements [30]. Also, dancers critically use decomposition techniques which consists in dividing a dance phrase into movement segments, for analyze and learn each segment [14]. Another challenge stems from learning in a classroom, which involves social interactions with other dancers and teacher. This contextual factor builds upon individual embodied knowledge to create complex learning strategies. In our previous works [36], we examined how dancers elicit these techniques and express their experiences. However, this work did not highlight operational mechanisms that could influence the design of technologies for supporting movement learning and movement transmission from teacher to dancers.

This paper examines how dancers approach the process of learning new movements and the potential of technology to uncover some of the tacit processes they follow. The first study explores how dancers report their learning process. This led to the analysis of how they structure their learning strategies by decomposing movements. To further explore this phenomenon, we created MoveOn, a technological tool to probe how dancers decompose video into short, repeatable clips, and saves a trace of the movement decomposition over time. We use MoveOn as part as a structured observation [23] during a second study where dancers learn a dance phrase from a video. In a third structured observation, we explored the use of the technology probe in movement transmission from a teacher to dancers. We compared two conditions: learning movement by following a movement decomposition created by a teacher versus learning the video phrase on their own. Finally, we discuss the benefits and limitations of our methodology, and we examine the social and collaborative issues observed during the workshops.

\section{RELATED WORK}

\subsection{Movement-based interactive systems for dance skill learning}

A common approach for supporting motor skill acquisition is to use a vision-based system to capture the users' movements and to augment the visual interface by displaying cues that help learning. For example, Anderson et al. [1] proposed YouMove, a Kinect-based system that provides guidance and feedback in order to help the user learn full body movements. A video on a screen displays the movement to perform and the user imitates it as closely as possible. The system offers guidance and feedback to instruct the user on which movement to perform and corrects them afterward. Compared to traditional video demonstrations, adding interactivity improves learning and short-term memory retention.

Bevilacqua et al. [5] propose a similar system called If/Then, which tracks and follows the user's gesture in real time to control videos of the choreographer Richard Siegal performing the "real" movement. These videos guide users as they learn the choreographer's vocabulary showing the correct way to perform the movements and encouraging them to explore the qualities of each dance movement, rather than simply imitating it. Further work by the same authors includes a pedagogical installation for learning movement qualities stemming from the vocabulary of choreographer Emio 
Greco. They showed that movement qualities can enhance the user experience [21] and be used as an efficient pedagogical cue [20].

Tsampounaris et al. [43] proposed a more immersive approach that places the user within a virtual reality environment that provides real-time feedback for the movement. To provoke selfreflection, they used avatars and visualizations in a gaming context that push the user to mimic kinetic material.

Although these systems are valuable for dance pedagogy, these systems do not reveal the operational details of how dancers learn movement. Instead, they focus on learning short movements, and do not require dancers to analyze and decompose whole phrases. Thus, it is unclear to what extent these systems can be used to learn more complex dance movements. Our goal is to better understand and support real-world situations where dancers acquire long dance phrases, with or without external cues.

\subsection{Dance movement annotation tools}

Annotation tools have the potential to enhance dance learning through personal augmentation of videos. Singh et al. [38] designed The Choreographer's notebook, a web-based application that allows choreographers and dancers to annotate videos, so they can collaborate and analyze rehearsal videos as a central artifact for discussion. They show how this increases efficiency of rehearsal time, helps learning, and enables online communication among dancers and the choreographer.

DanceNote, created by the company La fabrique de la danse, is a web tool that encourages choreographers and dancers to share, annotate, and learn from video content ${ }^{2}$. Dos Santos et al. [17] created a video annotation tool that lets dance teachers write comments or use predefined labels to assess a dance performance. Cabral et al. [7] designed an annotation tool that allows choreographers to add information on video and to share it with dancers. A later extension to a 3D environment allows choreographers to view and annotate video clips from multiple angles [35] Video Trace is a video annotation tool that lets teachers to annotate video clips while teaching dance composition and encourage students to reflect up on their work [11]. Ramadoss and Rajkumar [34] offer a semi-automated system for annotating and retrieving dance videos. Annotations are also an efficient way to classify, navigate and search in large multimedia dance databases [18, 22]. Ciolfi Felice et al. [13] designed Knotation, a tablet-based tool that lets choreographers create interactive sketches of their personal choreographic ideas with incorporated videos.

Although these annotation systems are useful for helping dance practitioners browse, retrieve, add personal information and share videos with other practitioners, none were designed to specifically tackle the problem of learning, nor to elicit how dancers learn movements.

\subsection{Designing in the context of the dance studio}

Dance movement learning is a social and collaborative human activity that benefits from investigation in real-world contexts [39], requiring methodological tools that inform various aspects around and beyond the technology [2].

In the larger CSCW community interested in learning, most of the methodologies involve the study of the users in the field with a commitment to understanding real-life learning tasks and their collaborative components [40]. Goodyear et al. [25] examined how to support sharing of professional knowledge within a geographically distributed community, arguing that this type of knowledge is partly tacit. They captured and shared such tacit knowledge by augmenting videoclip of users' practice with audio commentaries. In the dance studio, the socio-technical aspects of the Choreographer's Notebook was studied in three dance productions, in a field-based approach [9].

$\overline{{ }^{2} \text { http://www.lafabriquedeladanse.fr/dancenote/ }}$ 
They found an asymmetric use of the tool, where the choreographer provided the majority of the comments and the dancers posted questions. Although not specifically designed to elicit how dancers learn movements, they were able to investigate the pedagogical value of their design.

Molina et al. [33] created the Delay mirror which renders a video stream of the dancers in a classroom with a several second delay. This system allowed the dancers to analyze their own movements and the movements of their peers. A focus group investigated how the technology would be accepted in the context of a dance class, and how it allowed the dancers to comment collectively on their actions. However, this approach only provides an ephemeral trace of movements produced by one or several dancers, and does not investigate their learning strategies in this context.

Ciolfi Felice [12] used technology probes to design creativity-support tools for choreographers. Although not directly linked to learning, this approach allowed her to document phenomena beyond the design of the system itself and the creative process. The author identified three types of collaboration that emerged during the choreographic process: human-to-human collaboration, where dancers and choreographers are negotiating; collaboration between people and technology, where the technology promotes ideation and reflection; and collaboration among people through technology where the collaboration is synchronous or asynchronous. She suggests that Knotation can help the self-assessment of the students, and support the feedback given by the teacher.

In this paper, we adopt a grounded approach where dancers, in a dance studio, learn a dance phrase. We use a technology probe [28] to trace their learning and to provoke reflection and collaborative thinking about it.

\section{WORKSHOP 1: DOCUMENTING LEARNING TO EXTRACT DESIGN GUIDELINES}

Before designing new tools to explore dance learning, we need to better understand the practice schedule of dancers without interactive technology. We conducted a workshop with expert contemporary dancers where they captured their learning schedule on paper in order to:

(1) uncover dancers' learning strategies and observe how they report on it;

(2) identify the dancers' needs, the actions they performed, and the problems they encountered while learning dance from video.

The workshop asks dancers learn a solo dance sequence from a video recording while simultaneously document the way they practice the movements. We structured the documentation process according to the result of [36], where expert contemporary dancers engaged in the following learning techniques:

- Observation of the movement in its entirety or in details;

- Segmentation of the reference movement into smaller sequences;

- Mental simulation of the movement;

- Imitation of the movement in its entirety;

- Marking of the movement in a less than complete manner to work on a segment;

- Personal adaptation of the movement in order to make it easier to execute or more personal; and

- Repetition of one of the aforementioned learning strategies.

\subsection{Workshop structure}

3.1.1 Participants. We recruited 4 experienced contemporary dancers (all women, 2 professionals) with 6 to 26 years of experience, all with prior experience learning dance phrases from videos. Participants were invited to lunch or dinner as compensation for their participation. 
3.1.2 Setup and apparatus. The workshop was conducted in a dance studio in Paris, and lasted approximately two hours. Dancers viewed video on a tablet ${ }^{3}$ from a traditional video media player ${ }^{4}$. All participants agreed to participate in the study and signed a consent form validated by an ethics committee.

3.1.3 Procedure. The workshop comprises three parts. First, we present the research context and brief the participants on the upcoming tasks (20 minutes). We also performed a warm-up exercise where each participant created a movement associated with their name. All the movements created were repeated to form an exquisite corpse to introduce the participant of the workshop.

Second, participants were given one hour to learn a dance clip. The dance clip is an extract of one minute and forty-seven seconds solo from a dance piece call SKIN ${ }^{5}$, shot with a hand-held camera, performed by a professional contemporary dancer in a dance studio. The first 30 seconds of the video involve easy slow movements, and the second part involve more difficult fast movements with rotations and change of direction. We chose this video for its progressive difficulty, but also because it is a typical example of videos that dancers are expected to learn for dance auditions.

In parallel, we asked dancers to document the actions they did on the video player and the problems faced during learning by using the following supplies (see Figure 1):

- Card with the Learning technique names: observation, segmentation, mental simulation, imitation, marking, personal adaptation, repetition. Plus additional space for comments.

- Blank cards: let dancers propose new techniques.

- Pink Post-it notes: identify a problem encountered during the learning or the use of the video.

- Yellow Post-it notes: indicate the actions performed on the video.

- A0-size paper: allows dancers to place and, compose cards and Post-it notes.

In the following, we refer to the final paper artifacts created by participants as compositions. Importantly, the only requirement for participants was to document and report how they learned the phrase. We did not impose composition rules and let participants develop their own compositions and freely place the cards and post-its. In the last 30 minutes, we asked each participant to perform what they learned from the clip. We then asked them to explain their composition and engaged a group discussion about their learning process.

3.1.4 Data collection and analysis. We recorded video and audio of the session and took photographs. We also retrieved each participants' composition. The first author transcribed and analyzed the videos. Two authors iteratively analyzed the participants' compositions, videos and recordings using thematic analysis [26]. We defined concepts by using the participants' words (open coding) and grouped them into categories (axial coding). The analysis was verified and refined by a third author in order to converge into categories that best captured the data. Each of the authors involved in the analysis read the transcripts beforehand and was present during the study. The following data refer to the dancers as participant 1 to 4, denoted by P1 to P4.

\subsection{Results}

All four participants used the different learning techniques, added comments on the cards, and wrote (P1, P4) or drew (P3) on the A0 sheet. Participants learned between 0'42 (P3) and 1'02 (P2) of the proposed phrase.

3.2.1 Learning techniques in action. Three participants found learning technique cards sufficiently generic to successfully classify their actions. Yet, P3 and P4 used blank cards to create two new

\footnotetext{
${ }^{3}$ iPad apple iOS 6 version 10.3.3, screen $9.72048 \times 1536,14 \mathrm{G}$

${ }^{4}$ QuickTime

${ }^{5}$ http://saralaoui.com/2016/02/skin/
} 


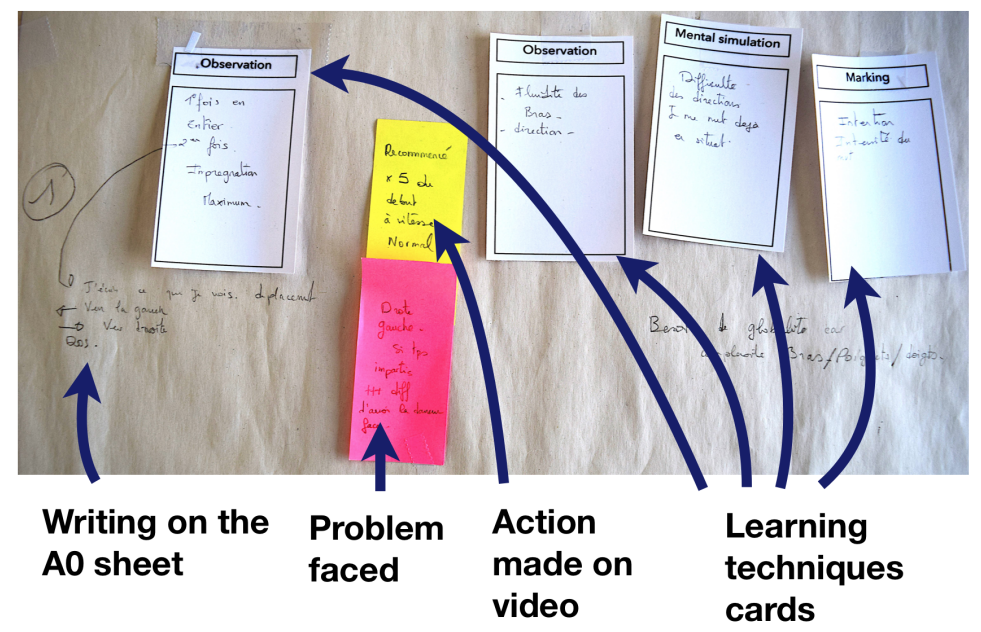

Fig. 1. Composition example: P1 annotated the learning techniques cards, added yellow Post-it notes to document the actions on the video and pink post-it notes to report the problems faced.

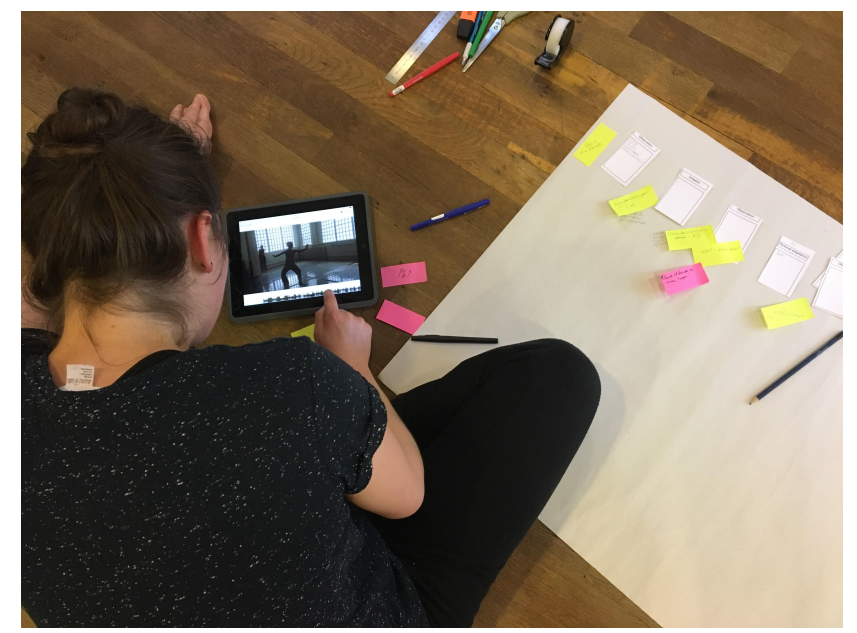

Fig. 2. Workshop 1: P1 interacts with the tablet to select the next movement to learn. Her composition on the right documents her learning process.

learning techniques. P3 created a draw card to capture the action of drawing the trajectory of a movement. P4 created a voice card for the action of verbalizing aloud. P1 said that the learning cards influenced her choices and that she would not have given these particular names to the techniques. We found that the learning techniques were used in a coupled manner rather than isolated. P3 used two repetition cards in her composition (see Figure 3.a), linked to the other cards by lines (see Figure 3.b): "There are inseparable things [...]. There are some techniques I would not have taken out. It is completely in repetition, everything works at the same time" (P3).

3.2.2 Movement decomposition. P2, P3 and P4's compositions identified movement segments with segmentation cards. P3 segmented the phrase into three parts: $0^{\prime}-0^{\prime} 32^{\prime \prime}$ : easy, $0^{\prime} 32$ " - $0^{\prime} 36^{\prime \prime}$ : transition, 


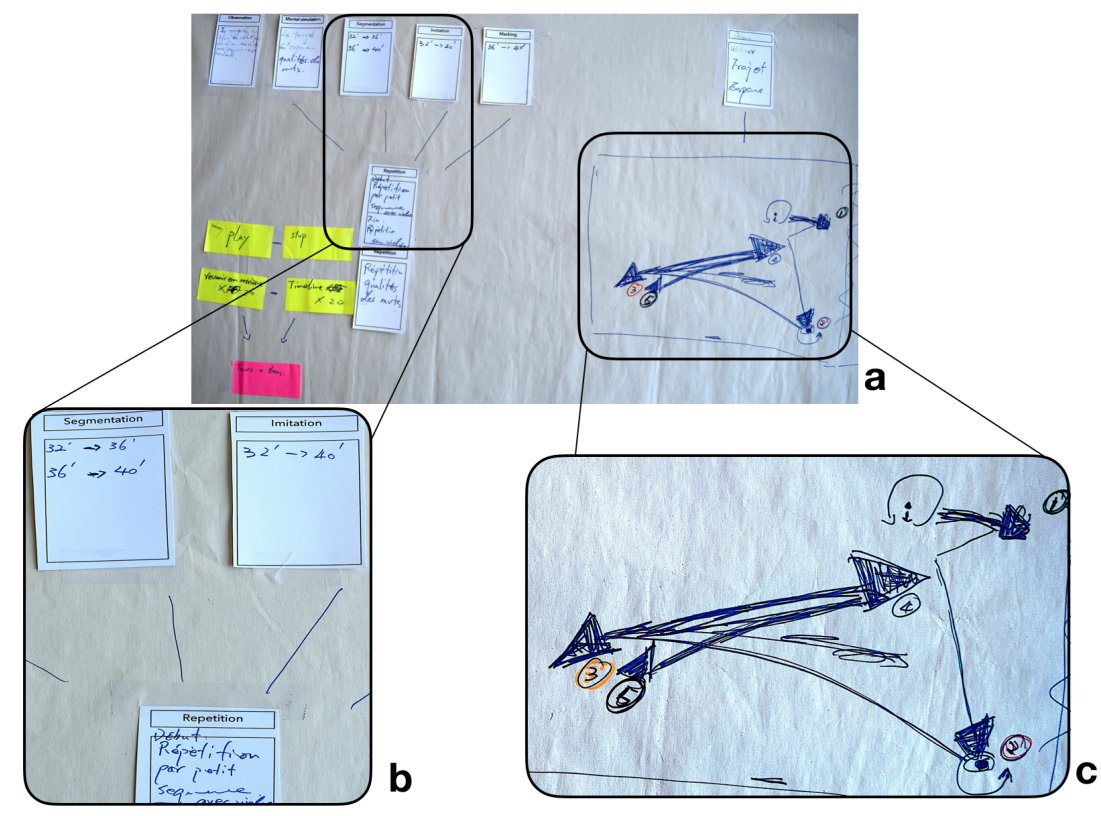

Fig. 3. Participant 3's composition: (a) Final composition composed of interconnected learning techniques (b) and drawings that show spatial displacements (c).

and $0^{\prime} 36^{\prime \prime}-0^{\prime} 40 "$ : complex movements. P4 and P2 segmented their learning into 2 parts. P2 indicated decomposing the movement when it became too complex while P4 indicated that she decomposed according to the diagonals, that refer to spatial markers of the movement.

P1 did not use segmentation's card in her composition. Her decomposition, however, include clusters of learning techniques, each starting with observation. For example: observation-repetitionpersonal adaptation then observation-mental simulation-marking-personal adaptation. These clusters identify the learning of the different parts of the phrase.

All dancers decompose the movement but for diverse reasons. On the one hand, P3 described her learning habits based on her previous experiences: "That's why at first I'll do it in more or less the same order. Based on my experience". P4 and P3 described their compositions as a summaries of their usual practice: "It's a little summary of my habits. When I work on choreography, I create things or learn things" (P3). Dancers also capture specific difficulties or elements of movement in their decomposition, such as the quality and the trajectory in space (P3, $\mathrm{P} 2)$, the motor of the movement (P1, P2) or the energy (P4).

3.2.3 Video interaction. We observed that their interactions with the video (yellow Post-it notes) are linked to the different learning techniques. Observation cards always follow or precede a yellow Post-it notes that indicates scrolling, playing or pausing the video. P3 also used a segmentation card to isolate a part that she named rotation+arms. She scrolled back this movement 20 times, to ensure she learned it . The action of scrolling back multiple times is then related to repeating a segmented part of the phrase.

All participants manipulated the progress bar in order to slow down movements: "I slowed down a lot, to come back, to see the trajectory, the arm, the supports" (P3). Although dragging the scroll-bar's thumb with the finger does slow the video, P1 and P2 found it jerky and ineffective: "While scrolling, 
you don't see energy, you don't see speed, time. And so I missed several period" (P2). Slowing down the movement seems linked to the decomposition of the movement and to the difficulty of the task.

Finally, the dancers were all frustrated by the limitations of the media player which forced them to interrupt their practice to play, pause or scroll the video: "I wasn't listening to my body going into space because I was watching the video and not experimenting" (P1). To avoid the back and forth between video control and dancing, all of the participant at one point, took the tablet in their hands and practiced the dance while handling it: "Having a pause button and dancing at the same time is not easy. Sometimes I danced with the tablet in my hand." (P1).

\section{CAPTURING MOVEMENT DECOMPOSITION WITH MOVEON}

In the first study, dancers used learning techniques cards, post-its and ntoes to report on their learning process. The created artifact made them reflect on their learning process. This reflection highlighted the structuring function of decomposition in the learning process.

However, technology-free documentation and group discussion could not bring more details on how dancers decompose movements. This led us to the design of a tool able to advance further in the analysis. We designed MoveOn, a technology probe [28] with the goal of capturing movement decomposition with two main features in mind: the ability to decompose a dance video into multiple interactive segments and saving a trace of this decomposition. In the following two sections, we elaborate the design of MoveOn.

\subsection{Decomposing into segments}

MoveOn allows the user to create segments. A segment is an interactive object that is associated with a part of a video. During the creation of a segment, the user can define the starting point and the duration of the segment. When a segment is created, it can be 'played', i.e. play the associated video part. This is depicted in Figure 4. To create a segment, the user needs to long press on the video scroll-bar's thumbs. This action makes a segment appears below the progress bar (see Figure 4.a) Then the user holds the long press and drags until the desired end point (see Figure 4.b). Once the user drops the progress bar, a segment is created below the video (see Figure 4.c).

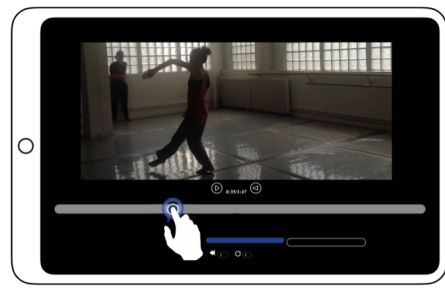

a) Long press to start creating a segment

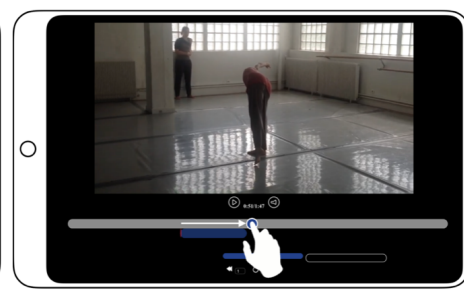

b) Drag to specify the size of the segment

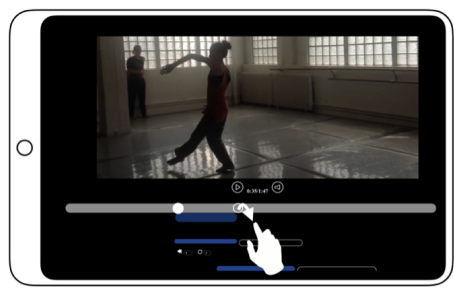

c) Drop to add the segment in the segment history

Fig. 4. There are three steps to create a segment: (a) first the user performs a long press on the video progress bar, to start the creation of a new segment. (b) While holding down, the user drags horizontally to increase or decrease the size of the segment. (c) When the user stops pressing, a new segment is created in the segment history below the video. When the user touches the segment, the corresponding video fragment is played.

In study 1 , we found that the learning techniques are not operating in isolation but rather are intertwined. We accounted for such inter-dependencies by enabling users to annotate, slow down or repeat the segment. Consequently, each segment allows the user to engage with the learning techniques of segmentation, repetition, observation, and imitation. In addition, we found in study 1 that repeated interactions with video interrupted the dancers in their practice. The segments allow 
the users to limit the number of interactions with the video by automatically performing actions they were performing manually such as repeating and slowing down.

Figure 5 depicts the visual aspect and the properties of a segment. For each segment, a user can define a number of repetitions, a speed and a text annotation. When the user touches a segment, the related part of the video is played the specified number of times at the defined speed. The visual representation of a segment embeds two properties: its starting point in the video (represented by its left margin) and its duration (represented by its length).

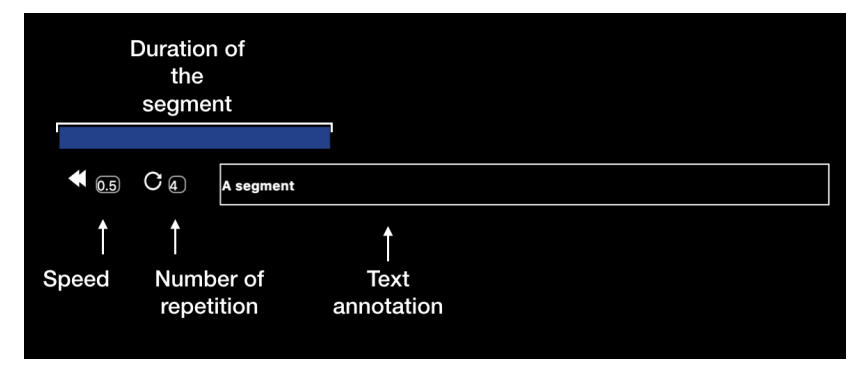

Fig. 5. A segment corresponds to a defined video part and embeds 3 properties: speed, number of repetitions and text annotation. Clicking on a segment will play the related video part the specified number of times at the defined speed.

\subsection{Tracing the decomposition through segments history}

Our second goal was to save a visual trace of the decomposition over time. The technology probe saves all the segments created by a user in a segment history. A segment history is a time-ordered stack of the different segments created by the user. A newly created segment is placed at the top of the segment history, so the first segment created is always at the bottom.

The creation of segments history retraces the dancers' learning pathway and decomposition. It is meant to enable the dancers to design and reflect on their learning process, and for the researchers to analyze and understand the dancers' decomposition strategies. The Figure 6 shows the entire interface of MoveOn.

\subsection{Implementation}

We created a web server in Node.js embedding a video media player. The web server allows for portability among several platforms: tablet for participants, and laptop for coding and debugging.

We recreated video controls from scratch to have total control over the features offered to the participants. We deliberately simplified the video's functionalities in comparison to a regular media player to reduce the possible interactions. The application allows users to play, pause or scroll the video, and to create or delete segments.

We implemented a save and load function of the segment history to be able to save and analyze the segment history of each participant after the session. We implemented an action logger that saves a trace of different actions performed on the website. We log the navigation actions in the video, playing or pausing a segment, changing its speed, and the number of repetitions for each segments. We chose to log these to reconstruct the actions performed with the probe for our future studies. 


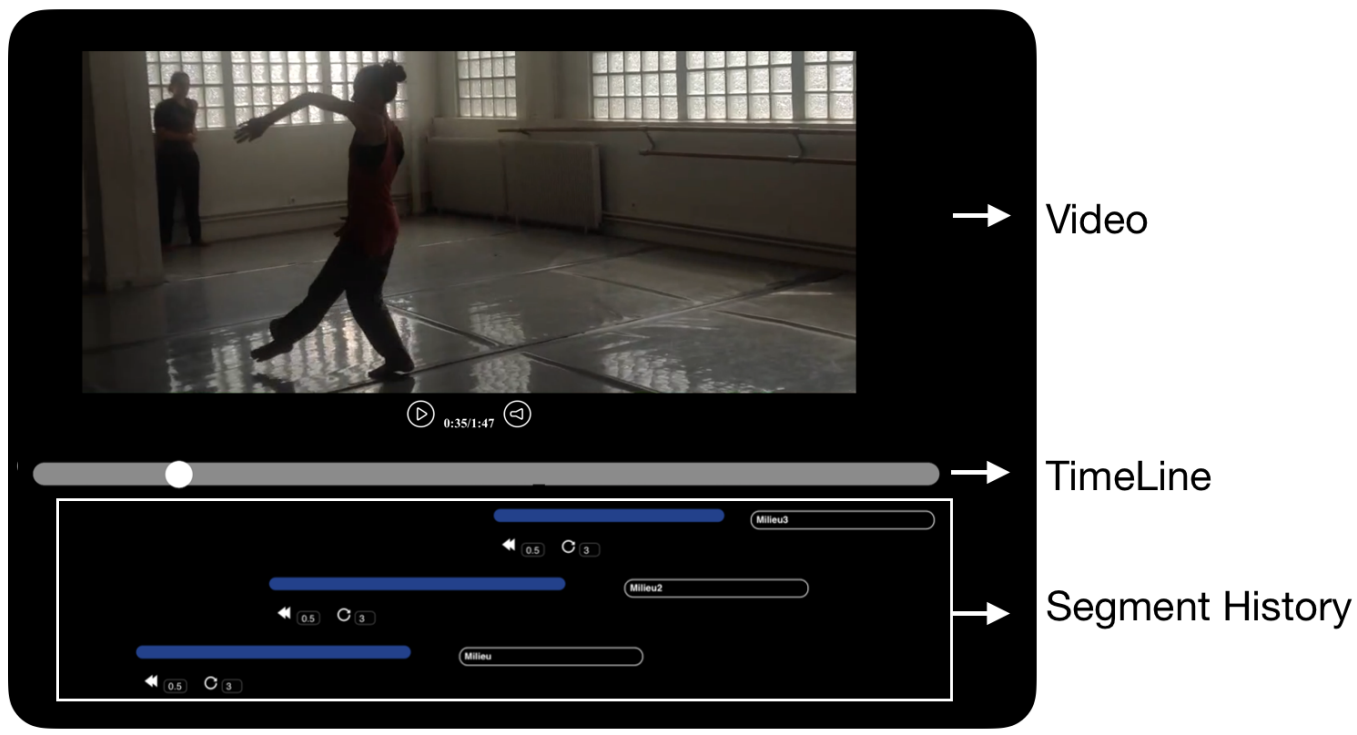

Fig. 6. The interface of the probe displays a dance video, the timeline of the video and a segment history containing three segments (in blue). The segment history has to be read from bottom to top. Each segment contains a speed-ratio and a repetition-number. The length of a segment corresponds to its duration, and the left margin to its positioning on the video.

\section{WORKSHOP 2: UNDERSTANDING THE DECOMPOSITION PROCESS}

In this second workshop, we aim to understand how dancers decompose a dance phrase while learning it, through the use of MoveOn introduced previously. We then use the decomposition produced to give them the opportunity to reflect on their learning process.

\subsection{Workshop structure}

5.1.1 Participants. We recruited 6 experienced contemporary dancers (5 women, 1 man) with 6 to 30 years of experience. The participants were recruited from our contacts and were not compensated but were invited for lunch before the session. All the participants reported having previous experience learning dance from videos. Two of the six participants recruited also participated in the first study described in Section 3. Study 2 took place one month after study 1.

5.1.2 Setup. We ran the session in a local dance studio. The session lasted approximately two hours. All participants agreed to participate in the study and signed a consent form validated by an ethics committee. Each participant was given a tablet ${ }^{6}$ with MoveOn installed and running, presenting the user with the same video used in the first workshop (see Section 3). Two laptops ${ }^{7}$ were used as servers to host the websites.

5.1.3 Procedure. We welcomed the participants to the studio and presented the general objective of our workshop. We described the task that consisted of learning the video-recorded dance excerpt while using the technology probe. We distributed the tablets and explained to the participants how

\footnotetext{
${ }^{6}$ iPad apple iOS 6 version 10.3.3, screen $9.72048 \times 1536,14 \mathrm{G} 6$

${ }^{7}$ MacBook Pro-macOS High Sierra, 13 inches, SSD 256Go, processor 2,3 GHz Intel Core i5, RAM 16Go and MSI GS60-Windows 7, 15 inches, processor 2,6 GHz Intel Core i5, RAM 8Go
} 
to start and stop the video, create segments, and interact with the created segments. We encouraged them to create multiple segments, we did not give them instructions about when and why they should create these segments. Once the technology was demonstrated, we gave the participants 45 minutes to learn the dance clip. We then asked each participant to perform what they learned from the clip. Finally, we sat altogether and asked each participant to explain their segment history. We engaged in a group discussion on the action of segmenting, the difficulty of the task and the opportunities and problems perceived.

5.1.4 Data collection and analysis. We documented the sessions through video recordings and photographs. We also logged all the segments created by the participants. The load function implemented in the probe (but not exposed to the participants) allowed us to visualize the complete segment histories, including deleted segments. This allowed qualitative comparison of the strategies of each participant. Two researchers iteratively analyzed participants' segments, segment histories, videos and recordings using thematic analysis [26]. Thematic analysis procedure followed was the same as that for Study 1 . In the following section, we anonymize the data and refer to the dancers as participants 1 to 6 , denoted P1 - P6.

\subsection{Results}

5.2.1 The use of the technology and the tablet. All participants created a segment history that included from 3 segments (P3) to 8 segments (P1, P2, P5, P6). P2 was the only one that created several segments before dancing, while the other participants created segments when they needed them. All participants at one point tried to hold the tablet in their hand and dance but they expressed that with the tablet, they felt constrained and could only mark the movement. Furthermore, P2 expressed that "it immobilized the arm holding the tablet". Instead, they alternated between interacting with the tablet in their hands or placing it on the floor so they could play the segments and dance at the same time, with or without looking at the screen.

5.2.2 Movement decomposition strategy. We identified two strategies when creating multiple segments: regrouping and ungrouping. On the one hand, regrouping corresponds to the creation of a new segment from multiple previously-created segments. P5 and P4 reported that they created segments that encompass several smaller segments. For example, P4 stated "I wanted to make a third one that includes the first two segments. To create a sequence, I created another one that included both". On the other hand, ungrouping correspond to exposing smaller chunks within a larger sequence. For instance, P6 started by creating a large segment and then dividing it into multiple smaller chunks: "I just make and forget the rest. Inside, if I perceived a strategic point, I would put back a little segment".

Regrouping and ungrouping strategies shed light on the ways in which the participants chose to handle segment transitions. The participants overlapped each segment with adjacent ones and repeated the transitions between each segment every time. P5 raised the possibility that the probe could be pro-active in handling segment transitions: "When you create a segment, it would be nice if it were directly at the end of the next one". Additionally, $\mathrm{P} 4$ raised a concern about a problem that could arrive from creating independent segments: "Between two dance sequences, there was some information I missed. Because it was neither in the end of one [segment] nor the beginning of the other".

5.2.3 Foci behind segment creation. Participants created segments according to various needs including when dance content was especially difficult or based on their personal interest surrounding a section of video content. P4 used segments to repeat part of the video and slowed it down in order to unravel the movement: "[Slow motion] helped me a lot looking at the details". P4 stated that the more rotation or speeds there were, the more she repeated or slowed down the movement. For 
P1, segmenting helped her figure out the "starting point of the movement" in order to reproduce the difficult movement.

We also identified various movement foci that drove the movement segmentation. Movements were segmented based on changes in characteristics such as space or the the starting point of the movement were used by participants to make decisions on their selection. For example, P5 segmented the phrase according to the spatial orientation: "The first sentence was because we were in front, I cut like this for the orientations".

5.2.4 Transmission and reuse. Collaborative discussion made participants suggest the potential of the technology to be used in movement transmission and pedagogy. P4 stated that segment histories are a way to "see the different approaches of people's segmentation". P4 would also like to see different annotation approaches: "It would be interesting to suggest some possibilities. Different ways, different annotation schemes". P6 suggested that beyond a personal context, MoveOn could be used for transmission: "Beyond memorization, I find it interesting for transmission. When I give a lesson, I film myself and I have to transmit it. It helps me get ready. How I am going to transmit it." She emphasized the potential of reusing one's composition: "when it's my movement, it is interesting to be able to say to the others, 'I'd like you to try to learn it this way, with this sequence". Additionally, P6 mentioned the probe's ability to share people's foci in dance: "We also have the concept of the person, on what he or she focuses on. That means what was important for him or her".

\section{WORKSHOP 3: EXPLORING DANCER-GENERATED VS TEACHER-GENERATED DECOMPOSITION}

In the previous workshop, we examined how dancers decompose a dance phrase from a video recording in order to learn it. We observed a variety of strategies used by dancers to decompose and recompose the movement. In workshop 3, we wanted to go further and explore the difference between the two typical pedagogical cases: self-scheduled learning by the dancer and learning from a schedule provided by a teacher.

\subsection{Workshop structure}

6.1.1 Design. The workshop is a qualitative inquiry of the impact of two decomposition strategies on learning a dance phrase from a video recording. First, we consider the teacher decomposition case where dance students learn a dance phrase using a movement decomposition previously created on the tool by a teacher. Second, we consider the dancer decomposition case in which dance students learn a dance phrase using their own self-created decomposition on the tool. The study followed a within-subjects design in which each participant experienced both cases of having to decompose the movement or to follow a pre-defined decomposition from a teacher.

6.1.2 Participants: dancers and teacher. The dance teacher that we collaborated with is a contemporary dance teacher from our university, with 40 years of teaching and dance experience. She teaches a contemporary dance class every week for 4 hours with 6 students. These 6 students are the 6 participants in our study. On average, the participants are dancing $4 \mathrm{~h}$ per week and practiced contemporary dance for 6 years. There are 5 female dancers and 1 male dancer, with an age range from 22 to 42 years old. None of the participants were involved in previous studies.

6.1.3 Videos and teacher decomposition. Before the study, we asked the teacher to choose two video clips of contemporary dance. We explicitly asked the teacher to choose two dance excerpts that her students had never seen before with equivalent levels of difficulty. The teacher chose two dance videos from the EAT (a national dance exam) that she clipped to be of equivalent length. The videos last 43 and 47 seconds respectively. Both videos are snippets of contemporary dance solos 
performed by professional dancers. These two videos vary in difficulty with relation to variations in movement just as variations in space, rotation, change of balance, falling and weights shifts. The links to the two original videos are reported in footnotes ${ }^{8}$.

For each video, the teacher created a decomposition of the dance phrase with the probe. We asked the teacher to: "decompose the video as you would teach it to your student. You can annotate segments, change the number of repetition and the speed. Remember that your student will have to strictly follow your decomposition to learn the dance phrase". We did not limit the time taken by the teacher to create these decompositions; she was free to explore and converge towards the most relevant decomposition pedagogically. She took approximately 4 hours to create the two decompositions (2 hours each).

6.1.4 Setup and procedure. We ran the session in a dance studio. The session lasted approximately two hours and half. Each participant was given an iPad tablet ${ }^{9}$ on which the tool was running on a web browser. Two laptops ${ }^{10}$ were brought in the dance studio and used as servers to host a local version of the website handling the video and logging.

Before the beginning of the workshop, all participants agreed to participate in the study and signed a consent form validated by an ethics committee. The participants were not compensated for their participation.

The dancers started by warming up for approximately 30 minutes, under the supervision of the teacher. Then we distributed the tablets and explained to the participants the use of the tool: how to start and stop the video, how to create a segment and how to interact with segments (i.e. clicking on the segment to play it, changing the number of repetitions, its playback speed and adding a label).

In the first activity, we asked the participants to learn a dance phrase from the video 1 using MoveOn on the tablet. We split the 6 dancers in 2 groups of 3 in a random manner. The first group had to learn the dance phrase from video 1 using their own decomposition on the probe. We encouraged them to create multiple segments, however we did not give them instructions about when or why to create a segment. The second group had to learn the same dance phrase using the decomposition made by the teacher and displayed on the probe. For this group, the instructions were: "Your goal is to learn the dance phrase on the video using the segmentation presented to you. You will start with the first segment (the one at the bottom) and play them one by one. You must let the segment play the number of times specified. When you finished following a segment, you can either continue to play it again or move on to the next segment."

Both groups had 30 minutes to accomplish this task. Afterward, we asked each participant to perform what they learned. We took a 10 minutes break to save each segment history and prepare the second activity.

In the second activity, we reversed the role. The first group followed the decomposition created by the teacher for the second video while the second group was instructed to learn the dance phrase from video 2 using by creating their own decomposition.

After 30 minutes, we also asked each participant to dance what they learned from the second video. After each performance, we distributed the same Likert scale questionnaire to the participants in order to evaluate their felt experience. This questionnaire inquired their satisfaction during the activity, their level of confidence in the acquisition of the phrase, their satisfaction over their

\footnotetext{
${ }^{8}$ Video 1: : https://www.numeridanse.tv/videotheque-danse/epreuves-de-danse-2015-danse-contemporaine-variation-11-fille Video 2: https://www.numeridanse.tv/videotheque-danse/epreuves-de-danse-2015-danse-ccontemporaine-variation-14-fille-1ere-option ${ }^{9}$ iPad apple iOS 6 version 10.3.3, screen $9.72048 \times 1536,14 \mathrm{G} 6$

${ }^{10}$ MacBook Pro-macOS High Sierra, 13 inches, SSD 256Go, processor 2,3 GHz Intel Core i5, RAM 16Go and MSI GS60Windows 7, 15 inches, processor 2,6 GHz Intel Core i5, RAM 8Go
} 
performance, the usefulness of the features (repeat, decompose, slowdown, annotate) and the helpfulness of the tool to analyze and understand the phrase and the practice.

Finally, in a group discussion, we asked each participant to reflect on and explain their segments history and we engaged in a dialogue on the differences between following an imposed decomposition versus creating their own, the difficulty of the task and the opportunities and problems perceived.

After the workshop, we conducted an interview with the teacher where we asked her to explain segment by segment the two decompositions that she created. Moreover, we asked her to evaluate the skills of the participants and to review their performances based on the captured videos of the session. She watched each performance and gave them a score from 1 to 10 .

6.1.5 Data collection and analysis. We documented the session through video recordings and photographs. We saved all the segment histories and logged all the actions performed by the participants (play, pause, navigating in the video with the progress bar, creating or modifying segments). Each participant's segment history was linked to their explanation. It was also linked to the teacher's rating of their skills and her scores of their performances. We evaluated the felt experience of the dancers through the Likert scale questionnaire and the statements made during the debriefing. We analyzed the round table, discussion and debriefing using thematic analysis [26] The procedure followed for the thematic analysis was the same as in workshop 1 . In the following, we will refer to the dancers as participants 1 to 6 , denoted $P 1-P 6$.

\subsection{Results}

6.2.1 Corroborating study 2. In both teacher and dancers decompositions, we found the same two strategies highlighted in Workshop 2 (see Section 5). More precisely, dancers and teacher decompose the movement by creating 2 to 3 shorter segments (that we called the ungroup strategy) and then re-assemble these segments into longer phrases (regroup strategy).

On managing the transition between neighbouring movements, we found that in both dancers or teacher decompositions, a newly created segment is almost always overlapping with the previously one created. In the two teacher decompositions, respectively $92 \%$ and $95 \%$ of the segments are overlapping with the previous neighbor. In dancers' decomposition, 4 dancers out of 5 generated more than $80 \%$ of overlapping segments, examples of overlapping segment are depicted in the Figure 8 and Figure 9, that represent respectively P1 and P2's decompositions.

The only exception is P3's decomposition where we can distinctly cluster four sets of segment that do not overlap. It seems that P3 worked on four distinct parts of the movement, without focusing on transitions. Interestingly, P3 did not succeed in performing the dance when using their personal decomposition, and this could be partly due to the fact that she did not include transitions in her decomposition.

6.2.2 Eliciting drives in movement decompositions between those created for learning and those created for transmission. Movement decomposition varies between dancers and teacher. Dancers created movement decomposition with the aim to learn the dance phrase in a limited amount of time. The teacher created movement decomposition with the aim to transmit the dance phrase to the student in the best possible way.

Regarding dancer decomposition, we confirmed certain foci used by the dancers and highlighted in the previous workshop. Foci that drive dancers' decompositions are mostly space and spatial orientation, completed with personal and idiosyncratic ones. All the dancers started decomposing from the beginning of the video, in a chronological order. Most of the participants (4/5) segmented the video in part according to the orientation of the movement in the scene (P2, P3, P4, P5). P2 segmented in that way in order to: "visualize and to know where I'm going in space. Once I know 
the direction, I can work on details". P5 also explained segmenting according to the dancer's static and dynamic movements. Interestingly, P1 chose to segment regardless of specific foci and created segments of equal duration (around 5 seconds). P1 explained that when he was facing a difficulty in the segmented part, he created a segment to zoom in on a difficult part. Indeed, creating segments to focus on a difficult part have been reported by more than half of the participant (3/5).

Regarding the teacher decomposition, we observed that they are far more elaborated than those of the dancers. She created 25 segments for the first video and 19 segments for the second video and spent 2 hours per decomposition. The teacher's decomposition of the first video is show in Figure 7. She created four types of segments, with different foci and goals that are as follows:

- Observational segments: observe the dance phrase and dance along with the video. These segments are characterized by a normal speed and one or two repetitions;

- Technical segments: specify technical information such as counting the steps or notify which leg starts;

- Spatial segments: cut the movement into space and orientation; and,

- Detail segments: detail specific difficult parts of the phrase. These segments are characterized by a short duration, a low speed and a high number of repetition.

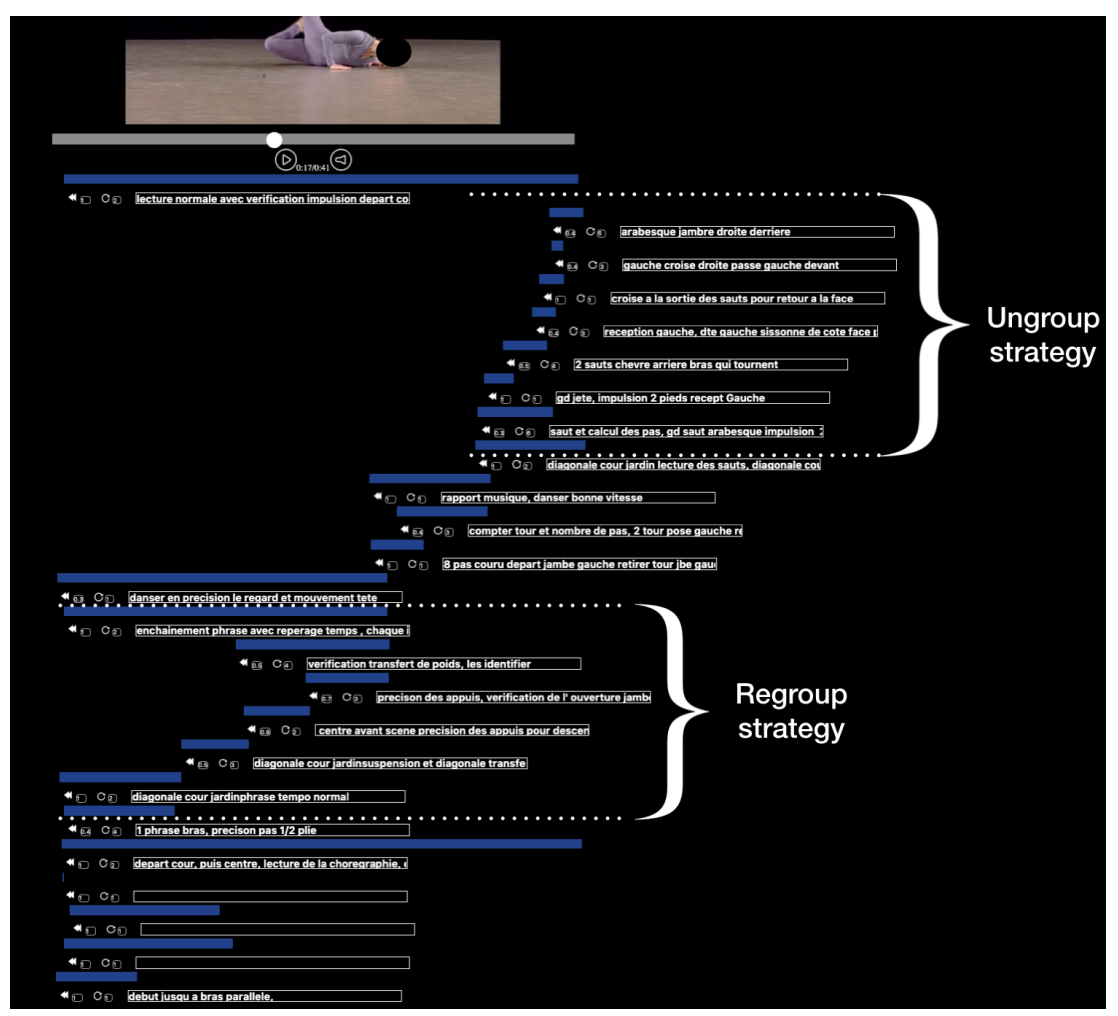

Fig. 7. The dancer teacher decomposed the first video into 25 segments overlapping segments. We detected the same strategies of regrouping and ungrouping segments in her decomposition.

6.2.3 Assessing dance performance after personal and imposed decomposition. To compare the learning using the teacher decomposition versus the dancer decomposition, we first report on the 


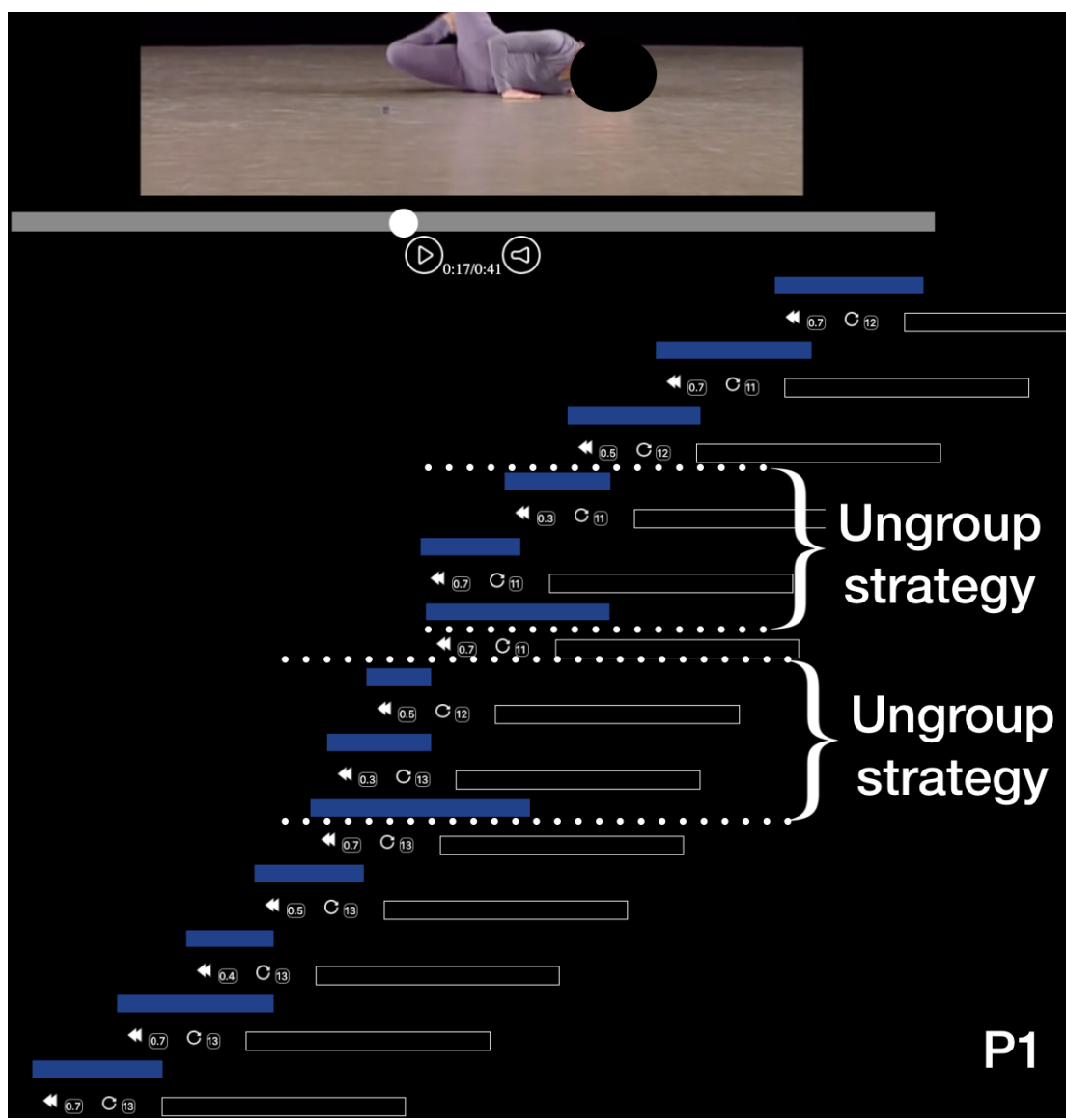

Fig. 8. The decomposition of P1 contains 13 overlapping segments. P1 used the ungroup strategy in order to exposes smaller chunks within a larger sequence.

scores provided by the teacher (after the workshop) on the dancers' expertise and the assessment of each of their individual performance.

According to the teacher, $\mathrm{P} 2$ is the most expert dancer and P3 is the least experienced dancer and never learned from a video before. P1, P4 and P5 are intermediate dancers. However, P4 explained facing difficulties when learning from a video: "for me, learning from a video is very complicated because the right and the left are reversed. (P4)". Using the teacher's decomposition, the two least expert dancers managed to perform and improve their learning of the dance. The teacher stated that P4's performance was "far more precise" and cleaner when she followed the teacher's decomposition. On the other hand, the most expert dancer (P2), performed the two dances exactly in both conditions but her learning was considered best when using her own decomposition. For the two intermediate dancers (P1 and P5), the teacher evaluated their two performances at the same level (with the teacher's decomposition P1 presented a slightly lower performance while P5 a slightly better performance).

6.2.4 Assessing dancers experience of personal and imposed decomposition. In terms of experience, the analysis of the Likert scales showed that all of the participants preferred their own decomposition 


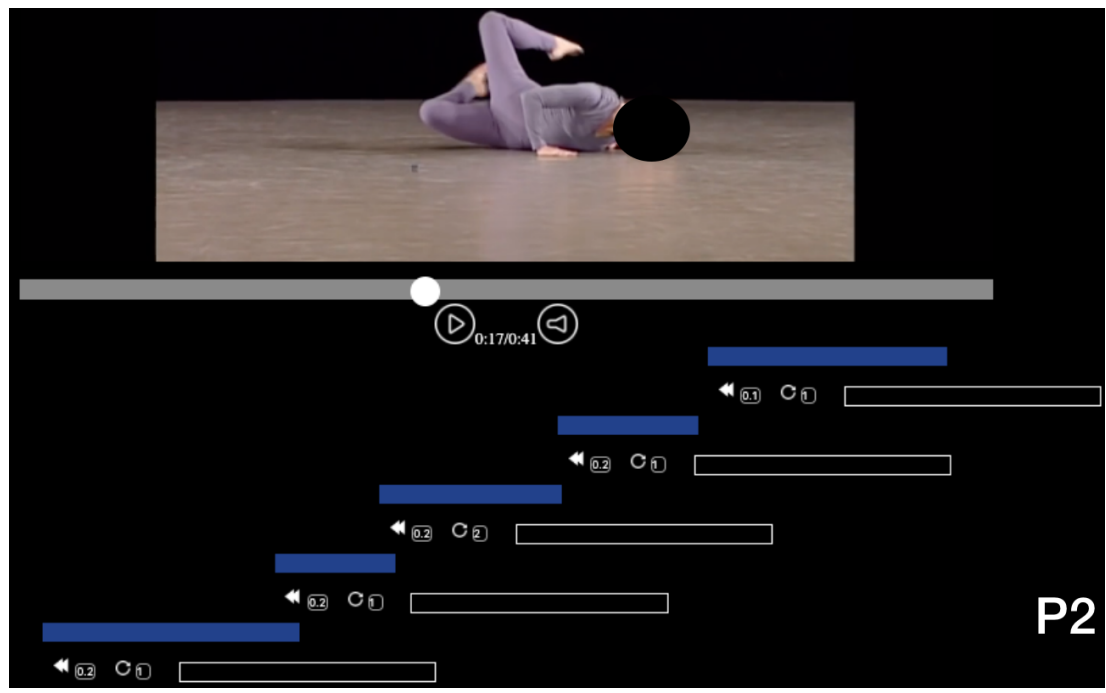

Fig. 9. The decomposition of P2, the most expert dancer of the session, contains 5 overlapping segments with a low speed rate (between $\mathrm{x} 0.1$ and $\mathrm{x} 0.2$ ).

compared to the teacher's decomposition. The dancers found that their own decomposition helped them learn the dance phrase. When asked to rate the features of the interface with respect to the task, they stated that the lowering speed and repetition functions were useful for learning. Only P3, the most novice dancer, stated that the teacher's decomposition suited her because it was "segmented with a lot of detail for the same sequence" (P3). This is consistent with the fact that P3 performed better with the teacher's decomposition.

We identified two problems related to the teacher's decomposition that may have an impact on the dancers experience: the lack of choice and a confusion over the different segments. Most of the participant (4/5) expressed frustration when following the teacher's decomposition segment by segment. According to P2, that feeling was emphasized when starting the session with the system in autonomy and then getting constrained in the second activity. This lack of choice was particularly felt when the intention of dancers conflicted with that of the teacher. P2 argue that the logic of the decomposition was different and P5 and P1 stated that they got interrupted in their observation because the focus of the teacher was not the same than theirs: "and it cuts me right in the middle of what I was looking" (P5). Moreover, most of the participants (4/5) did not understand the intentions behind the teacher's decomposition. Although the teacher annotated all the segments, P1 argued that he did not understand what he was supposed to look at.

\section{DISCUSSION}

In this paper, we first show how dancers, when asked to learn from a video, structure their learning strategies by decomposing movements. From these first observations, we design a web-based tool that lets dancers decompose video into short, repeatable clips to support their learning. Using this tool, we probed for changes in focus, decomposition and recomposition strategies adopted by dancers when learning a dance phrase or by a teacher willing to transmit a dance phrase. We found consistent strategies in movement decomposition but with different foci of attention depending on the dancer's difficulties or the teacher's pedagogical intentions. Our results suggest that teacher-imposed decomposition is more effective for introductory dance students, whereas 
personal decomposition is more suitable for expert dancers. Here, we discuss these findings, propose implications for designing technology to support analysis, learning and teaching of dance through movement decomposition. Finally, we discuss the impact of the context, as a social factor, on our results.

\subsection{Movement decomposition using MoveOn as a lense}

The strength of the methodology used, based on technology probes [28] and first-person perspective design [27], is to inspire us to reify users' actions into interaction techniques. The process of reification is defined as "the process by which concepts are turned into objects $[3,4]$. In the context of this work, the concept of reification allowed us to transform dancers' learning techniques (e.g repetition, segmentation, movement foci among others) into concrete interactive objects. To be more precise, in MoveOn, a segment of video reifies the concept of segmenting and repeating a chunk of the dance choreography at a certain speed, several times. Through reification, we give the user more agency via the possibility of manipulating concrete visible tools that embody their practice. This also allows us to reveal and visually render the user's habitual methods. By making it interactive, we open opportunities for new methods and know-hows to emerge.

The use of the tool by the dancers made decomposition strategies apparent. In other words, the segment history made the learning schemes adopted by each dancer more tangible. Interestingly, the resulting segment histories elicited distinguishable patterns. There seems to be consistency in the way dancers decompose a dance phrase in relation to where the segments are placed, in which order as well as how segments are combined or divided. While the amount of data collected in the second and third workshops is too limited to further analyze those patterns, the fact that a tendency can already be observed with a limited number of dancers is promising. Further research would benefit from a larger sample of dancers by deploying the system more widely. This would certainly bring new insights on movement decomposition, beyond strategies found in this paper and consequently advance the knowledge in more theoretical aspects of motor learning such as chunking, which is the decomposition of sequences of movements into strongly associated sub-segments $[15,24]$.

\subsection{MoveOn as a technology probe between movement learning and transmission}

On the one hand, designing learning schemes is a fundamental problem in the field of motor skill acquisition. Several works have addressed this problem by looking at the ways to schedule tasks and their variations during practice [37]. This strategy has been tested in various cases such as implicit learning [44], sport practice [42], or music [8], with varying results according to the field of application. The proposed tool may provide new perspectives on analyzing learning schedules and extracting prototypical learning schemes. Although this is not explored in this work, we believe that such feature would be important to highlight in future work.

On the other hand, designing learning schemes is also a fundamental problem in pedagogy. The proposed tool has the potential to serve as a medium between the teacher and dancers for instance in distributed or remote learning. From our results, we suggest that while less experienced dancers performed better in the imposed decomposition, they still prefer learning with their decomposition. This result support previous findings that learning practices leading to better performance are not necessarily preferred by learners $[10,31]$. Therefore, there seems to be a compromise to reach, between the condition in which they perform best and the one they prefer. One approach could be the combination of both modes: a curated movement decomposition that can be easily customizable by the learner. Currently we are not fully aware of all the phenomena that impact the felt experience and the level of success of a task [6] but we highlighted significant starting points to explore this question. 
For intermediate or expert dancers, however, following the teacher decomposition seems ineffective. Our interpretation is that, through extensive practice, advanced dancers create functioning learning patterns that they can reuse across tasks [19]. Yet, their own decomposition still helps them to visualize and reflect on their learning process. Reusing their own decomposition opens numerous questions such as how can we effectively reuse or share similar patterns across dancers or videos?

\subsection{Social and collaborative nature of the dance studio}

Dance studio is a complex social environment involving explicit and implicit interactions between dancers, dancer-teacher and dancer-technology. This environment makes it hard to understand the impact of the group on the use of MoveOn and its impact on dancers' practice. Nonetheless, we observed social and collaborative aspects of learning that contribute to $\mathrm{HCI}$ and $\mathrm{CSCW}$ in particular.

7.3.1 Group discussions. During the group discussion of the second workshop we showed that one aspect that has been commented by dancers is the extent to which movement decomposition and segment history could be shared across them, or could be used as a transmission tool. This drove the design of the follow-up workshop where dancers were learning through their own decomposition or the teacher's decomposition. Results were mitigated. As reported, imposed decomposition may help novices but less experts. At a social level, previous works showed that, in a computer-mediated learning context, teachers play an important role in facilitating technology adoption by the students $[16,40]$. In our context, facilitation seems to be facing strong individual-specific drives, such as the criteria used to decide when the movement segments start or stop. In response to the dancers' group discussion in workshop two, this challenges the shareability of segment history in the context of learning. More research is thus needed to better understand the trade-off between sharing learning schedules and supporting the idiosyncratic nature of motor skill learning.

7.3.2 Social context. The paper has reported on a series of three workshops that took place at different moments with different groups of dancers. An important contextual factor has been the difference between groups from workshops 1, 2 and workshop 3. In workshops 1 and 2, the participants were all intermediate or expert dancers with experience in learning dance choreography from video. They were recruited among our contacts, and were highly motivated to participate to the study. In workshop 3, the study took place in a dance class, with heterogeneous expertise among participants: two dancers were novices without prior experience on learning from dance video, and one participant was a professional dancer with an extensive experience of learning from video. During this workshop, we observed unexpected reactions triggered by the task. Mainly, a dancer abandoned the workshop before the end of the task.

This led us to question and challenge our methodological approach. Our original approach in this work was to consider a first-person perspective as a tool to generate design ideas. This approach brought insightful knowledge on learning strategies and techniques used by dancers during practice (as reported in the three workshops). However, it also pushes dancers to learn the dance movement on their own, using a tactile device. Dancers during workshop 2 were accommodating to the task, which we believe is due to their original interest in being part of these experiments. Dancers during workshop 3 were more resistant to the approach. For example, the dancer who was not able to finish the task further explained that the task reminded her of traumas encountered in childhood dance classes, where she had trouble to learn dance phrases. This highlights a conflict between the embodied and social nature of dance learning (in a studio) and the isolated nature of the technology supporting learning, as it has been designed. Therefore, while learning is an idiosyncratic process, 
taking into consideration group social dynamics, context, histories, and embodied practices are critical to support learning through technology.

\section{CONCLUSION AND FUTURE WORK}

In this paper, we presented three workshops that investigated how dancers describe the process of learning new movements from a video, adopting a first-person perspective to drive the design of a technology probe called MoveOn, that is assessed in a grounded valid context.

In a first study, we reported on how dancers document their practice using a framework from our previous research. Based on these insights, we designed MoveOn, a technology probe that allows dancers to reify subsets of the video into interactive segments and segments histories. We assessed this system in a second workshop with dancers learning a dance phrase from a video and in a third workshop, comparing how dancers learn from their own decomposition versus from that of their teacher using our probe. From the history tracing dancers' practice, we discovered strategies that dancers followed to create segments, manage transitions, and shift from movement details to higher level choreographic themes. We also suggested that following the decomposition of a teacher can offer benefits to novice dancers.

Therefore, our results contribute to understanding how dancers approached the problem of learning movements, more specifically how they decompose and recompose dance segments as they learn and how transferable a decomposition is from a teacher to a student. Furthermore our results highlight how the social context consisting of a group of dancers in a dance studio can facilitate dancers' reflection on their practice but can also raise tensions within the group. In this way, our work provides insights for future research in the design of technology to support the learning of complex corporal activities.

\section{ACKNOWLEDGMENTS}

This work was partially supported by European Research Council (ERC) grants № 321135 “CREATIV: Creating Co-Adaptive Human-Computer Partnerships”; the European Union's Horizon 2020 research and innovation program under the Marie Skłodowska-Curie fellowship (H2020) grant agreement № 659232, and the Agence National de la Recherche (ANR) grants № ANR-18-CE330002 "Enabling Learnability in Embodied Movement Interaction" (ELEMENT). We thank all the participants in our studies for their involvement.

\section{REFERENCES}

[1] Fraser Anderson, Tovi Grossman, Justin Matejka, and George Fitzmaurice. 2013. YouMove: Enhancing Movement Training with an Augmented Reality Mirror. (2013), 311-320. https://doi.org/10.1145/2501988.2502045

[2] Liam J Bannon and Kjeld Schmidt. 1989. CSCW: Four characters in search of a context. In ECSCW 1989: Proceedings of the First European Conference on Computer Supported Cooperative Work. Computer Sciences Company, London. https://doi.org/10.7146/dpb.v18i289.6667

[3] Michel Beaudouin-Lafon. 2000. Instrumental Interaction: An Interaction Model for Designing post-WIMP User Interfaces. In Proceedings of the SIGCHI Conference on Human Factors in Computing Systems (CHI '00). ACM, New York, NY, USA, 446-453. https://doi.org/10.1145/332040.332473

[4] Michel Beaudouin-Lafon and Wendy E Mackay. 2000. Reification, polymorphism and reuse: three principles for designing visual interfaces. In Proceedings of the working conference on Advanced visual interfaces. ACM, 102-109. https://doi.org/10.1145/345513.345267

[5] Frédéric Bevilacqua, Norbert Schnell, and Sarah Fdili Alaoui. 2011. Gesture capture: Paradigms in interactive music/dance systems. In Emerging Bodies, transcript Verlag, G. Klein, and S. Noeth (Eds.). 183-193. https: //hal.archives- ouvertes.fr/hal-01106962 cote interne IRCAM: Bevilacqua11a.

[6] Robert A Bjork, John Dunlosky, and Nate Kornell. 2013. Self-regulated learning: Beliefs, techniques, and illusions. Annual review of psychology 64 (2013), 417-444. https://doi.org/10.1146/annurev-psych-113011-143823 
[7] Diogo Cabral, Urândia Carvalho, João Silva, João Valente, Carla Fernandes, and Nuno Correia. 2011. Multimodal Video Annotation for Contemporary Dance Creation. In CHI '11 Extended Abstracts on Human Factors in Computing Systems (CHI EA '11). ACM, New York, NY, USA, 2293-2298. https://doi.org/10.1145/1979742.1979930

[8] Baptiste Caramiaux, Frédéric Bevilacqua, Marcelo M. Wanderley, and Caroline Palmer. 2018. Dissociable effects of practice variability on learning motor and timing skills. PLOS ONE 13, 3 (03 2018), 1-18. https://doi.org/10.1371/ journal.pone. 0193580

[9] Erin A. Carroll, Danielle Lottridge, Celine Latulipe, Vikash Singh, and Melissa Word. 2012. Bodies in Critique: A Technological Intervention in the Dance Production Process. In Proceedings of the ACM 2012 Conference on Computer Supported Cooperative Work (CSCW '12). ACM, New York, NY, USA, 705-714. https://doi.org/10.1145/2145204.2145311

[10] Christine E. Carter and Jessica A. Grahn. 2016. Optimizing Music Learning: Exploring How Blocked and Interleaved Practice Schedules Affect Advanced Performance. Frontiers in Psychology 7 (2016), 1251. https://doi.org/10.3389/fpsyg 2016.01251

[11] Gina Cherry, Janice Fournier, and Reed Stevens. 2003. Using a digital video annotation tool to teach dance composition. Interactive Multimedia Electronic fournal of Computer - Enhanced Learning 5 (1 12 2003).

[12] Marianela Ciolfi Felice. 2018. Supporting expert creative practice. Theses. Université Paris-Saclay. https://tel. archives-ouvertes.fr/tel-01984888

[13] Marianela Ciolfi Felice, Sarah Fdili Alaoui, and Wendy E. Mackay. 2018. Knotation: Exploring and Documenting Choreographic Processes. In Proceedings of the 2018 CHI Conference on Human Factors in Computing Systems (CHI '18) ACM, New York, NY, USA, Article 448, 12 pages. https://doi.org/10.1145/3173574.3174022

[14] Scott deLahunta and Philip Barnard. 2005. What's in a Phrase? Tanz im Kopf/Dance and Cognition (2005), 253-266.

[15] Jörn Diedrichsen and Katja Kornysheva. 2015. Motor skill learning between selection and execution. Trends in cognitive sciences 19, 4 (2015), 227-233. https://doi.org/10.1016/j.tics.2015.02.003

[16] Brian Dorn, Larissa B. Schroeder, and Adam Stankiewicz. 2015. Piloting TrACE: Exploring Spatiotemporal Anchored Collaboration in Asynchronous Learning. In Proceedings of the 18th ACM Conference on Computer Supported Cooperative Work; Social Computing (CSCW '15). ACM, New York, NY, USA, 393-403. https://doi.org/10.1145/2675133.2675178

[17] Augusto Dias Pereira dos Santos, Lian Loke, and Roberto Martinez-Maldonado. 2018. Exploring Video Annotation As a Tool to Support Dance Teaching. (2018), 448-452. https://doi.org/10.1145/3292147.3292194

[18] Katerina El Raheb, Aristotelis Kasomoulis, Akrivi Katifori, Marianna Rezkalla, and Yannis Ioannidis. 2018. A Web-based System for Annotation of Dance Multimodal Recordings by Dance Practitioners and Experts. , Article 8 (2018), 8 pages. https://doi.org/10.1145/3212721.3212722

[19] K Anders Ericsson, Ralf T Krampe, and Clemens Tesch-Römer. 1993. The role of deliberate practice in the acquisition of expert performance. Psychological review 100, 3 (1993), 363. https://doi.org/10.1037//0033-295X.100.3.363

[20] Sarah Fdili Alaoui, Frederic Bevilacqua, and Christian Jacquemin. 2015. Interactive Visuals As Metaphors for Dance Movement Qualities. ACM Trans. Interact. Intell. Syst. 5, 3, Article 13 (Sept. 2015), 13:1-13:24 pages. https://doi.org/10. 1145/2738219

[21] Sarah Fdili Alaoui, Baptiste Caramiaux, Marcos Serrano, and Frédéric Bevilacqua. 2012. Movement Qualities As Interaction Modality. In Proceedings of the Designing Interactive Systems Conference (DIS '12). ACM, New York, NY, USA, 761-769. https://doi.org/10.1145/2317956.2318071

[22] William Forsythe and S deLahunta. 2011. Motion bank. Motion Bank (2011).

[23] Jérémie Garcia, Theophanis Tsandilas, Carlos Agon, and Wendy E. Mackay. 2014. Structured Observation with Polyphony: A Multifaceted Tool for Studying Music Composition. In Proceedings of the 2014 Conference on Designing Interactive Systems (DIS '14). ACM, New York, NY, USA, 199-208. https://doi.org/10.1145/2598510.2598512

[24] Fernand Gobet, Peter C.R. Lane, Steve Croker, Peter C-H. Cheng, Gary Jones, Iain Oliver, and Julian M. Pine. 2001 Chunking mechanisms in human learning. Trends in Cognitive Sciences 5, 6 (2001), 236 - 243. https://doi.org/10.1016/ S1364-6613(00)01662-4

[25] Peter Goodyear and Christine Steeples. 1998. Creating shareable representations of practice. ALT-7 6, 3 (1998), 16-23. https://doi.org/10.1080/0968776980060303

[26] Greg Guest, Kathleen M MacQueen, and Emily E Namey. 2011. Applied thematic analysis. Sage Publications.

[27] Kristina Höök, Baptiste Caramiaux, Cumhur Erkut, Jodi Forlizzi, Nassrin Hajinejad, Michael Haller, Caroline Hummels, Katherine Isbister, Martin Jonsson, George Khut, et al. 2018. Embracing First-Person Perspectives in Soma-Based Design. In Informatics, Vol. 5. Multidisciplinary Digital Publishing Institute, 8. https://doi.org/10.3390/informatics5010008

[28] Hilary Hutchinson, Wendy Mackay, Bo Westerlund, Benjamin B. Bederson, Allison Druin, Catherine Plaisant, Michel Beaudouin-Lafon, Stéphane Conversy, Helen Evans, Heiko Hansen, Nicolas Roussel, and Björn Eiderbäck. 2003. Technology Probes: Inspiring Design for and with Families. In Proceedings of the SIGCHI Conference on Human Factors in Computing Systems (CHI '03). ACM, New York, NY, USA, 17-24. https://doi.org/10.1145/642611.642616

[29] David Kirsh. 2009. Knowledge, Explicit Vs Implicit. Oxford Companion to Consciousness (2009), 397-402. 
[30] David Kirsh. 2013. Embodied Cognition and the Magical Future of Interaction Design. ACM Trans. Comput.-Hum. Interact. 20, 1, Article 3 (April 2013), 30 pages. https://doi.org/10.1145/2442106.2442109

[31] Nate Kornell and Robert A Bjork. 2008. Learning concepts and categories: Is spacing the "enemy of induction"? Psychological science 19, 6 (2008), 585-592. https://doi.org/10.1111/j.1467-9280.2008.02127.x

[32] Richard A Magill and Kellie G Hall. 1990. A review of the contextual interference effect in motor skill acquisition. Human movement science 9, 3-5 (1990), 241-289. https://doi.org/10.1016/0167-9457(90)90005-X

[33] Luis Molina-Tanco, Carmen García-Berdonés, and Arcadio Reyes-Lecuona. 2017. The Delay Mirror: A Technological Innovation Specific to the Dance Studio. In Proceedings of the 4th International Conference on Movement Computing (MOCO '17). ACM, New York, NY, USA, Article 9, 6 pages. https://doi.org/10.1145/3077981.3078033

[34] Balakrishnan Ramadoss and Kannan Rajkumar. 2007. Semi-automated annotation and retrieval of dance media objects. Cybern. Syst. 38, 4 (April 2007), 349-379. https://doi.org/10.1080/01969720701291189

[35] Claudia Ribeiro, Rafael Kuffner, Carla Fernandes, and João Pereira. 2016. 3D Annotation in Contemporary Dance: Enhancing the Creation-Tool Video Annotator. In Proceedings of the 3rd International Symposium on Movement and Computing (MOCO '16). ACM, New York, NY, USA, Article 41, 4 pages. https://doi.org/10.1145/2948910.2948961

[36] Jean-Philippe Rivière, Sarah Fdili Alaoui, Baptiste Caramiaux, and Wendy E. Mackay. 2018. How Do Dancers Learn To Dance?: A First-person Perspective of Dance Acquisition by Expert Contemporary Dancers. In Proceedings of the 5th International Conference on Movement and Computing (MOCO '18). ACM, New York, NY, USA, Article 6, 7 pages. https://doi.org/10.1145/3212721.3212723

[37] John B Shea and Robyn L Morgan. 1979. Contextual interference effects on the acquisition, retention, and transfer of a motor skill. Journal of Experimental psychology: Human Learning and memory 5, 2 (1979), 179. https://doi.org/10.1037/ 0278-7393.5.2.179

[38] Vikash Singh, Celine Latulipe, Erin Carroll, and Danielle Lottridge. 2011. The Choreographer's Notebook: A Video Annotation System for Dancers and Choreographers. In Proceedings of the 8th ACM Conference on Creativity and Cognition (C\&C '11). ACM, New York, NY, USA, 197-206. https://doi.org/10.1145/2069618.2069653

[39] Anu Sööt and Ele Viskus. 2014. Contemporary approaches to dance pedagogy-The challenges of the 21st century. Procedia-Social and Behavioral Sciences 112 (2014), 290-299. https://doi.org/10.1016/j.sbspro.2014.01.1167

[40] Gerry Stahl, Timothy Koschmann, and Daniel D. Suthers. 2005. Computer-Supported Collaborative Learning. Cambridge University Press, 409-426. https://doi.org/10.1017/CBO9780511816833.025

[41] Shogo Tanaka. 2013. The notion of embodied knowledge and its range. encyclopaideia 37 (2013), 47-66.

[42] Antonios K Travlos. 2010. Specificity and variability of practice, and contextual interference in acquisition and transfer of an underhand volleyball serve. Perceptual and motor skills 110, 1 (2010), 298-312. https://doi.org/10.2466/PMS.110.1. 298-312

[43] Georgios Tsampounaris, Katerina El Raheb, Vivi Katifori, and Yannis Ioannidis. 2016. Exploring Visualizations in Real-time Motion Capture for Dance Education. In Proceedings of the 20th Pan-Hellenic Conference on Informatics (PCI '16). ACM, New York, NY, USA, Article 76, 6 pages. https://doi.org/10.1145/3003733.3003811

[44] Gabriele Wulf and Richard A Schmidt. 1997. Variability of practice and implicit motor learning. Fournal of Experimental Psychology: Learning, Memory, and Cognition 23, 4 (1997), 987. https://doi.org/10.1037/0278-7393.23.4.987

Received April 2019; revised June 2019; accepted August 2019 